\title{
Mindestmengen in der Chirurgie
}

\author{
Johannes O. Jost ${ }^{\mathrm{a}}$ Ernst Klar \\ a Klinik für Allgemein-, Viszeral- und Gefäßchirurgie, Franziskus Hospital gGmbH, Bielefeld, \\ ${ }^{b}$ Abteilung für Allgemeine, Thorax-, Gefäß- und Transplantationschirugie, Chirurgische Klinik und Poliklinik, \\ Universität Rostock, Deutschland
}

Am 1. Januar 2004 hat der Gesetzgeber mit dem $§ 137$ Abs. 1 Satz 3 SGB V eine Mindestmengenverordnung erlassen. Ziel war die Sicherung einer hochwertigen, humanen und wirtschaftlichen Patientenversorgung. Von Anfang an ist die Mindestmengenvereinbarung, insbesondere auf ärztlicher Seite, sehr kritisch gesehen worden, weil die wissenschaftlichen Daten für den Zusammenhang von Menge und Qualität unsicher waren. Daraus erfolgte eine rechtliche Unsicherheit in der Frage, wie mit Krankenhäusern umzugehen sei, die sich an diese Vorschriften nicht halten würden.

Die erste, von den Selbstverwaltungsgremien im Gesundheitswesen konsentierte, Mindestmengenvereinbarung umfasste 5 operative Eingriffe (Nieren-, Leber- und Stammzellentransplantationen sowie komplexe Eingriffe am Organsystem Pankreas und Ösophagus. Für Pankreas und Ösophaguseingriffe wurden zunächst fünf Operationen pro Jahr vereinbart. Im Jahre 2006 wurde diese Zahl verdoppelt und zusätzlich eine Mindestmenge für die Implantation einer Kniegelenksendoprothese eingeführt. Auch hier fehlte eine ausreichende Evidenz, die eine solche Verdoppelung der bestehenden Mindestmenge hätte wissenschaftlich absichern können. Daher wurde aufgrund der Anregung der Leistungserbringer und der Patientenvertreter im gemeinsamen Bundesausschuss die ursprüngliche Mindestmengenvereinbarung um den Auftrag zu einer wissenschaftlichen Begleitforschung ergänzt, die von Dezember 2005 bis zum November 2007 durch ein unabhängiges Forscherkonsortium aus drei Düsseldorfer Instituten durchgeführt wurde. Die Evaluation der Daten führte zu folgenden Empfehlungen:

1. Die Qualitätssicherung bei Transplantationen sollte nicht mittels Mindestmengenvorgaben durchgeführt werden.

2. Bei Ösophagus- und Pankreaseingriffen wird die Reduktion der festgesetzten Mindestmenge auf 5 pro Krankenhaus und Jahr empfohlen, um die sogenannte Gelegenheitschirurgie einzuschränken, ohne eine zu starke Einschränkung in der flächendeckenden Versorgung zu bewirken.

3. Die Mindestmengenhöhe für Knieprothesen wird als adäquat eingestuft.

4. Vor Einführung weiterer Mindestmengen wird eine modellhafte Überprüfung der Auswirkung auf die flächendeckende Versorgung sowie eine systematische Entwicklung empfohlen.

Die Diskussion um die Bedeutung einer Mindestmengenvereinbarung wird zwar derzeit nicht mehr so aufgeregt geführt, aber nach wie vor kontrovers behandelt. Aus diesem Grunde hat sich die Schriftleitung der Zeitschrift CHIRURGISCHE GASTROENTEROLOGIE INTERDISZIPLINÄR entschlossen, mit einem Diskussionsforum zu wesentlichen Fragen der Mindestmengenproblematik Stellung zu beziehen. Als Resümee ergibt sich, dass eine Mindestmengenvereinbarung allein sicherlich nicht die gewünschte Qualitätsverbesserung im Gesundheitswesen erbringen wird. Gute Qualität ist von zahlreichen weiteren Faktoren abhängig; eine Mindestmengenfestlegung kann in diesem Kontext eine sinnvolle Bereicherung der Maßnahmen sein. Sicherlich ist der Aspekt nicht zu vernachlässigen, dass ohne Mindestmengen eine Qualitätssicherung mit validen Aussagen nur eingeschränkt möglich ist.

Zusammenfassend konnte nach den Ergebnissen der vorliegenden Begleitforschung festgestellt werden, dass durch die Mindestmengenverordnung die an sie gestellten Erwartungen im Hinblick auf eine deutliche Qualitätsverbesserung nicht erfüllt werden können. Gleichwohl hat sie einen hohen Stellenwert im Bereich der Qualitätssicherung chirurgischen Handelns.

\section{KARGER}

Fax +497614520714

Information@Karger.de

www.karger.com
() 2008 S. Karger GmbH, Freiburg

Accessible online at:

www.karger.com/cga
Prof. Dr. med. Johannes O. Jost

Klinik für Allgemein-, Viszeral- und Gefäßchirurgie

Franziskus Hospital gGmbH

Kiskerstraße 26, 33615 Bielefeld, Deutschland

Tel. +49 521589 19-01, Fax -04

JostJO@aol.com 